Mechanobiologie

\title{
Zelluläres Tauziehen: Wie Zellen auf mechanischen Stress antworten
}

KAI WEISSENBRUCH, MARC HIPPLER UND MARTIN BASTMEYER

ZOOLOGISCHES INSTITUT, ZELL- UND NEUROBIOLOGIE, KARLSRUHER INSTITUT FÜR

TECHNOLOGIE (KIT)

\section{The ability of cells to sense and respond to extracellular forces is critical for cellular and tissue homeostasis. Tension or compression act on our body ubiquitously and cells respond to such mechanical cues by producing intracellular forces on their own. In this article, we briefly highlight the cellular and physical basis driving these pheno- mena and discuss our recent technical advance to stimulate and monitor the cellular mechanoresponse on a molecular scale.}

DOI: $10.1007 / \mathrm{s} 12268-021-1598-0$

(C) Die Autoren 2021

Das physikalische Wechselwirkungsprinzip ist allgegenwärtig im Leben, ob beim Sport, beim Anheben der Kaffeetasse oder beim Autofahren. Körperliche Anstrengungen - und seien sie noch so klein - erzeugen eine biomechanische Gegenreaktion. Unser Körper ist in der Lage, den erforderlichen Aufwand für die zu erbringende Arbeit wahrzunehmen und generiert als Antwort eine Reaktionskraft, die abhängig von der Intensität des physikalischen Stimulus ist. Dieses Prinzip lässt sich bis zu unseren mikroskopischen Funktionseinheiten, den Zellen, herunterskalieren. Das prominenteste Beispiel hierfür ist die Kontraktion von Muskelgeweben: Jede makroskopisch ausgeführte Kraftanstrengung resultiert aus einer hochkomplexen Orchestrierung mikroskopischer Kräfte, die von Millionen einzelner Zellen generiert werden. Zunehmend wird jedoch auch die zentrale Rolle intrazellulärer Kräfte in nichtmuskulären Zellen, wie Fibroblasten oder Epithelzellen, erkannt. Fast jeder Zelltyp unseres Körpers ist dazu fähig, nicht nur die biochemischen, sondern auch die mechanischen Gegebenheiten der extrazellulären Umgebung (ECM) wahrzunehmen. Diese zelluläre Mechanorezeption beinhaltet u. a. auch die Wahrnehmung einwirkender Kräfte, wie beispielsweise Zugspannungen und Kompressionen [1]. Entsprechend einem Wechselwirkungsprinzip reagiert die Zelle auf einwirkende Kräfte mit der Anpassung eigener Kräfte. Intrazelluläre Kräfte sind ein grundlegender Baustein für viele entwick-
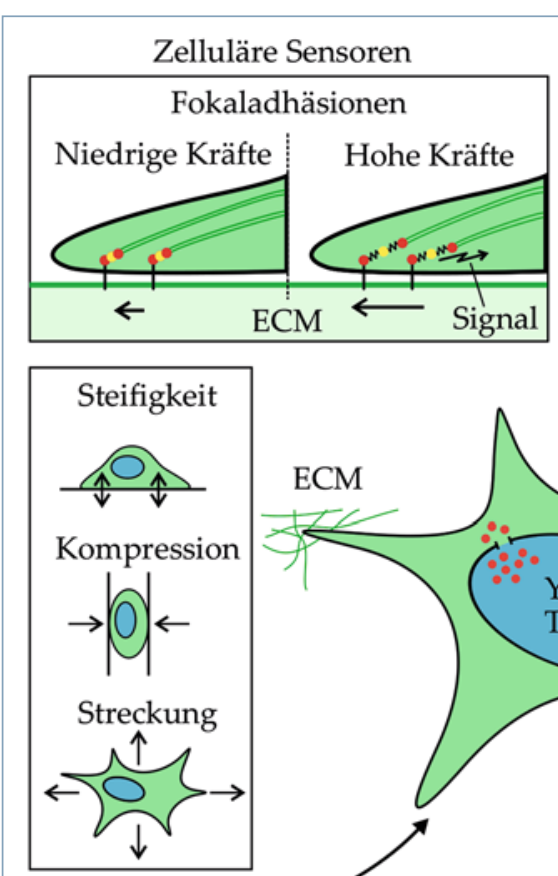

Mechanischer Impuls Ko-Aktivatoren. lungsbiologische und (patho)physiologische Prozesse [2]. Die Ausbildung stabiler Zelladhäsionen, die Reorganisation der Zellmorphologie während der Zellmigration oder die Cytokinese sind nur einige Beispiele, die in hohem Maße von einem raumzeitlichen Aufbau intrazellulärer Kräfte abhängig sind. Wie das letztlich im Detail abläuft, weiß allerdings noch niemand so richtig.

Molekulare Mechanismen der Mechanorezeption und -transduktion

Die molekularbiologischen Mechanismen der Rezeption, Konvertierung und Transduktion extrazellulärer Kräfte sowie die Erzeugung intrazellulärer Kräfte (Abb. 1) haben großes Interesse in der Forschung hervorgerufen. Die meisten Zellen stehen in permanentem

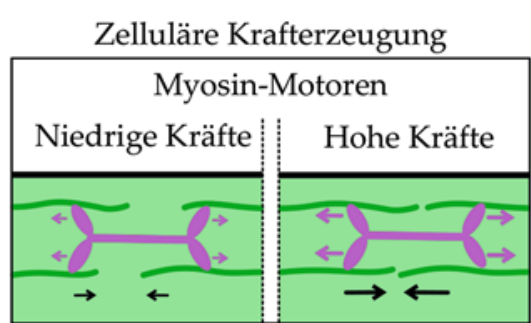

AktomyosinKontraktilität

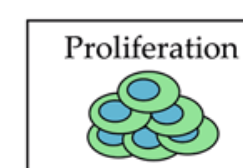

Migration<smiles>c1ccccc1</smiles>

Differenzierung
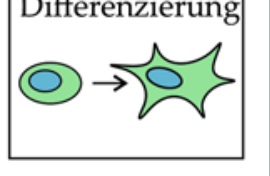

Interaktion und Remodellierung Feedback
Biologische Antwort

A Abb. 1: Mechanotransduktion in nichtmuskulären Zellen. Extrazelluläre mechanische Impulse, wie Streckungen oder Kompressionen, werden über transmembrane zelluläre Sensoren wahrgenommen. Die Signaltransduktion umfasst die Konvertierung des Impulses im Cytoplasma und dessen Weiterleitung bis in den Zellkern. Als Reaktion werden intrazelluläre Kräfte generiert, die an der biologischen Antwort beteiligt sind. ECM: Extrazelluläre Matrix; YAP/TAZ: transkriptionelle 
Abb. 2: Rasterelektronenmikroskopische Aufnahme einzelner Mikrostrukturen ohne Hydrogel (A, B). C, Die kolorierte Zelle verdeutlicht die selektive Zelladhäsion an den Balken und es ist zu erkennen, dass zelluläre Kräfte die Wände der Mikrostruktur nach innen biegen. D, Da das Hydrogel nicht für die elektronenmikroskopische Probenpräparation geeignet ist, wird dessen Funktion schematisch dargestellt.
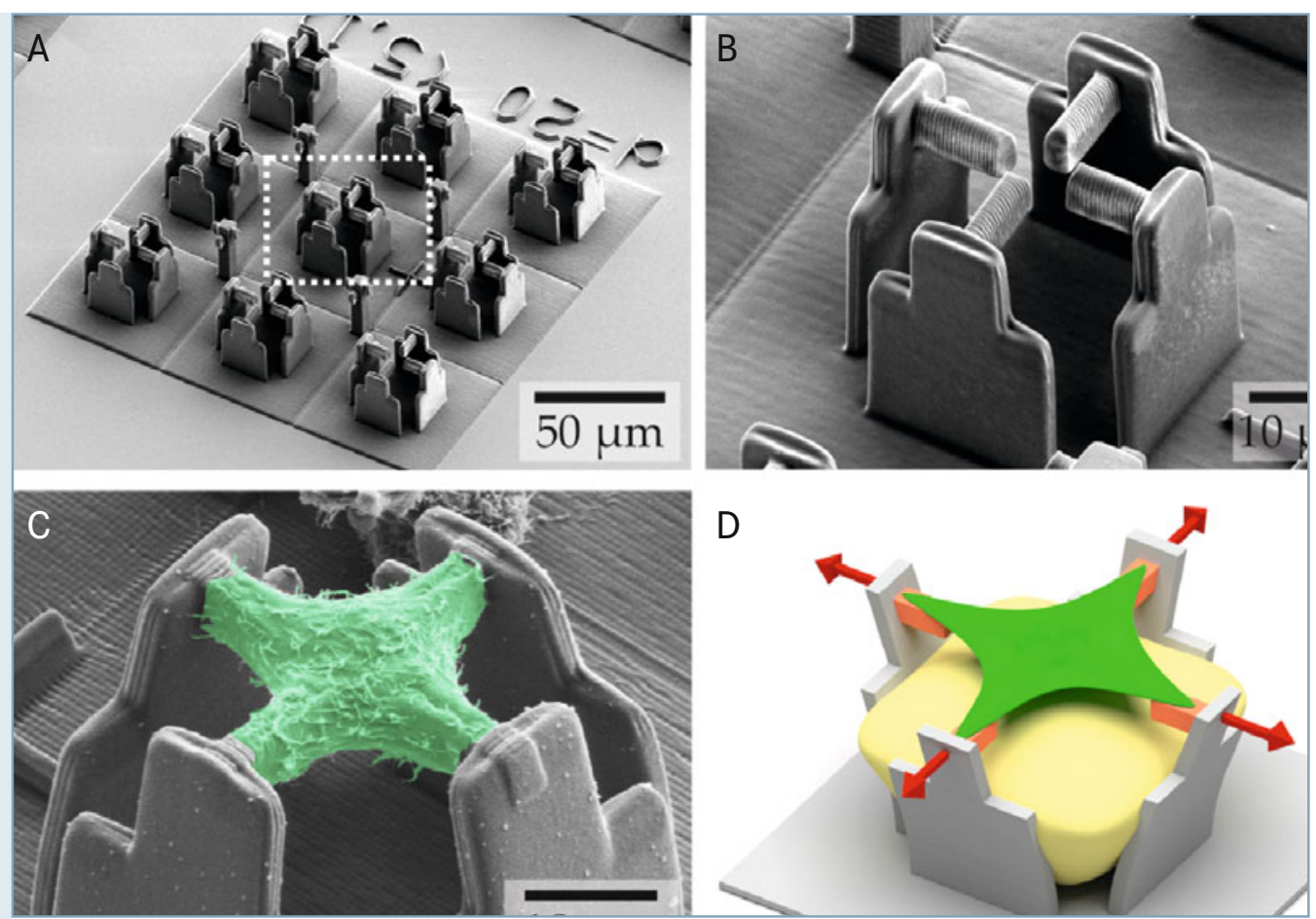

sensorischem Kontakt mit ihrer Umgebung. Auf der Zelloberfläche fungieren Adhäsionskomplexe wie Fokalkontakte als transmembrane Sensoren und vermitteln die mechanische Signalrezeption zwischen Zelle und ECM [3]. Intrazellulär sind die Sensoren an das Aktin-Cytoskelett gekoppelt, wodurch das Signal weitergeleitet und in eine biochemische Antwort konvertiert wird. Abhängig von der Intensität des mechanischen Stimulus manifestiert sich das Signal auf verschiedenen Systemebenen. Im Cytoplasma induzieren Zugspannungen die Freilegung von Proteindomänen an Fokalkontakten, ähnlich dem Prinzip einer elastischen Feder. Das ermöglicht die Bindung intrazellulärer Adaptorproteine an den Zielmolekülen sowie die Steuerung der Aktivität durch Phosphorylierungen [4]. Im Zellkern manifestiert sich die Mechanotransduktion auf transkriptioneller Ebene. Starke Zugspannungen fördern beispielsweise den Transport der transkriptionellen Ko-Aktivatoren YAP/TAZ in den Nukleus und begünstigen die Expression bestimmter Gene [5]. Aus übergeordneter biologischer Sicht kann eine solche Signalkaskade die Migration, Proliferation oder Differenzierung einer oder mehrerer Zellen auslösen. Aus mechanischer Sicht beinhalten diese Prozesse eine Anpassung der zellulären Kontraktilität. Die Grundlage hierfür bilden hochdynamische Interaktionen von Aktinfilamenten und molekularen Motorproteinen der Myosin-Klasse II. Unter Verbrauch von ATP können diese Proteine Kräfte in Pikonewton(pN)-Bereich aufbauen [6]. Zum Vergleich: Um ein Gewicht von einem Gramm überhaupt anzuheben, muss etwa eine Kraft von 10.000.000.000 pN erbracht werden. Eine Vielzahl an Myosin-II-Proteinen generiert in der Gesamtheit die Kraft, welche von einzelnen Zellen aufgebracht werden kann. Da die Kopfdomänen der Myosine quasi permanent mit den Aktinfilamenten assoziiert sind, übertragen sich die Kräfte wiederum auf die sensorischen Zelladhäsionen und es entsteht eine positive Rückkopplung. So kann präzise abgeleitet werden, ob es beispielsweise energetisch günstiger ist, die extrazelluläre Matrix zu remodellieren oder an eine andere Position zu migrieren.

\section{Die Problematik der experimentellen Analyse}

Dass wir trotz des relativ guten Verständnisses der molekularen Bausteine immer noch so wenig über zelluläre Kräfte und die Mechanotransduktion wissen, hat auch damit zu tun, dass die Methoden zur Stimulierung, Erfassung und Messung zellulärer Kräfte äußerst feinfühlig und präzise arbeiten müssen. Die Skala der zellulären Kontraktilität bewegt sich, je nach Zelltyp, im Bereich von Nanonewton (nN) bis Mikronewton $(\mu \mathrm{N})$ [7]. Extrazelluläre Kräfte, die auf die Zellen einwirken, bewegen sich ebenfalls auf sehr niedrigen Skalen. Um die Zellen mechanisch zu stimulieren, muss eine Kraft aufgebracht werden, welche zwar eine Zellantwort auslöst, jedoch niedrig genug ist, um die zelluläre Integrität zu wahren. Typischerweise werden Zellen dafür auf einem elastischen Material kultiviert, das gestreckt oder komprimiert werden kann. In der Praxis haben sich Membranen und PDMS-Oberflächen bewährt, die mit motorisierten oder Piezo-gesteuerten Elementen deformiert werden [8]. Will man beides kombinieren, also stimulieren und gleichzeitig die zellulären Kräfte messen, wird es noch anspruchsvoller. Ansätze hierfür umfassen u. a. optische Pinzetten und die Rasterkraftmikroskopie (AFM) [9]. Grundlage dieser Methoden ist, dass das Messinstrument gleichzeitig als kraftausübendes und als sensorisches Element fungiert. Bei optischen Pinzetten verwendet man dafür adhäsive Mikrokügelchen, die sich im Fokus eines Laserstrahls befinden und beim AFM eine als cantilever bekannte Messnadel. Beide Methoden bestechen dadurch, dass sie äußerst sensitiv und gezielt Kräfte auf subzellulären Skalen ausüben und die zelluläre Reaktion auslesen können. Der Nachteil ist jedoch, dass sie sequenziell und sehr zeitaufwendig sind, weshalb in der Regel nur eine Zelle pro Ansatz analysiert werden kann.

\section{Mini-Streckbänke für Einzelzellen}

Wir haben eine Methodik entwickelt, mit der es möglich ist, die Mechanosensitivität von vielen Einzelzellen simultan, mit hoher 
Hier steht eine Anzeige.

Springer 


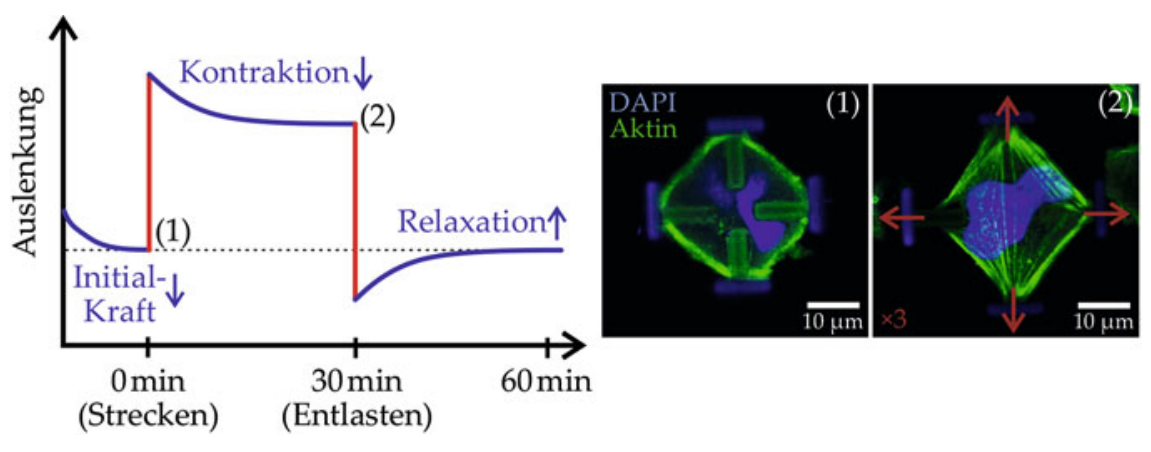

$\Delta$ Abb. 3: Auslenkungen der adhäsiven Balken dienen als Referenz für die Messreihe. Diese werden entweder durch intrazelluläre Kräfte ausgelöst (blau) oder durch Stimulation des Hydrogels (rot). Die fluoreszenzmikroskopischen Aufnahmen korrespondieren mit dem Ausgangszustand (1) sowie dem gestreckten Zustand (2) der Zelle und verdeutlichen die Verstärkung der Aktinfasern durch die mechanische Streckung.

Präzision und geometrischer Ordnung, anzuregen und gleichzeitig die zelluläre Kontraktilität zu messen [10]. Der Ansatz beruht auf der Kombination eines 3D-Nanodruckverfahrens mit einem stimulierbaren Material. Mithilfe des 3D-Nanodrucks ist es möglich, dreidimensionale Zellsubstrate mit quasi beliebiger Geometrie auf der Mikrometerskala und darunter herzustellen (Abb. 2A, B, [11]). Die „Drucktinten“ sind in diesem Fall Fotolacke, die im Fokus eines Femtosekunden-gepulsten Lasers polymerisieren. Indem unterschiedliche Fotolacke mit hydrophoben oder hydrophilen Eigenschaften verwendet werden, lassen sich mikrostrukturierte 3D-Gerüste mit proteinadsorbierenden und proteinabweisenden Bereichen herstellen. Dadurch kann die Zelladhäsion räumlich gesteuert und die geometrische Krafteinwirkung präzise vorhergesagt werden. Im aktuellen Fall werden drei verschiedene Fotolacke verwendet. Das Grundgerüst der Mikrostruktur besteht aus vier Wänden mit proteinabweisende Eigenschaften. An den Spitzen der Wände befinden sich vier horizontale Balken mit proteinadsorbierenden Eigenschaften, welche die Zelladhäsion vermitteln (Abb. 2C). Das stimulierbare Herzstück der Mikrostruktur bildet ein Hydrogel, das inmitten der Wände platziert ist und auf einem Wirt-Gast-System basiert. Die Wirt- und Gast-Moleküle sind an das Rückgrat des Acrylamid-basierten Hydrogels gebunden und haben eine starke Bindeaffinität zueinander, was zu einer Quervernetzung der Polymerketten führt. Wirt-Gast-Interaktionen befinden sich dabei zunächst im Equilibrium, wobei sich Bindungen durch Zugabe von kompetitiven, gelösten Gast-Molekülen öffnen können. Das Hydrogel kann in Folge mehr Wasser aufnehmen und schwillt innerhalb von
Sekunden an. Das zunehmende Volumen drückt die Wände der Mikrostruktur auseinander und die adhärente Zelle wird mechanisch gestreckt (Abb. 2D). Dieser Vorgang ist komplett reversibel, sodass theoretisch beliebig viele Zyklen der mechanischen Stimulation durchgeführt werden können. Der Aufbau der erwarteten intrazellulären Kräfte wird in Zeitrafferaufnahmen verfolgt, wobei die Bewegung der adhäsiven Balken als Messskala dient. Kennt man die Federkonstante, kann das hookesche Gesetz angewendet werden und die Distanzänderungen lassen sich in Kräfte übersetzen. Wie sich in unseren Experimenten zeigte, sind die Strukturen flexibel genug, um zelluläre Kräfte im Nanonewton-Bereich zu messen. Auf einer typischen Probe befinden sich bis zu 300 einzelne Mikrostrukturen auf einem Deckglas, was bedeutet, dass sich eine Vielzahl von Einzelzellen gleichzeitig stimulieren lässt. Da die Gast-Moleküle zudem in den gängigen Zellkulturmedien und Puffern löslich sind, können die Zellen jederzeit im gestreckten Zustand fixiert und die molekularen Bestandteile immunhistochemisch gefärbt werden. Somit lässt sich eine eingängige, molekularbiologische Analyse an das Experiment anschließen.

\section{Mechanische Homöostase: Wie Zellen auf der Streckbank reagieren}

Wir haben dieses System benutzt, um in ersten Experimenten die Mechanosensitivität von U2OS-Zellen zu testen (Abb. 3). Wie sich zeigte, generieren diese Zellen bereits im Ausgangszustand, also allein durch die Zelladhäsion, eine „Initialkraft“ von ca. $70 \mathrm{nN}$. Durch die mechanische Streckung verdoppelte sich diese Kraft innerhalb von 30 Minuten auf ca. 140 nN. Nach diesem Zeitraum hatte sich die Kontraktilität auf ein neues Niveau eingependelt. Wird der mechanische Zug gelöst, reduzieren die Zellen ihre Kräfte bereits nach wenigen Minuten wieder auf das Ausgangsniveau. Die Zellen sind also in der Lage, ihre intrazellulären Kräfte in Abhängigkeit der äußeren Kräfte in kürzester Zeit zu regulieren - ein Prozess, der in der Literatur unter dem Begriff „mechanische Homöostase" erwähnt wird [12]. Wie erkennen Zellen, was die ideale Kraftbalance in bestimmten Situationen ist? Welche Moleküle dienen hier als Sensor und welche als Verstärker? Bemerkenswert ist, dass bereits das Fehlen eines einzelnen Proteins, dem nichtmuskulären Myosin IIA (NM IIA), ausreichend ist, um die komplette Maschinerie zum Erliegen zu bringen. Zellen, in denen kein NM IIA mehr exprimiert wird, besaßen in unseren Experimenten weder eine messbare Initialkraft, noch zeigten sie eine Reaktion auf den mechanischen Stress. Immunhistochemische Färbungen der gestreckten Zellen zeigten, dass die Zellen zwar ihre Fläche vergrößern und die Aktinfasern partiell verstärken, die intrazellulären Kräfte jedoch nicht an die neuen Bedingungen angepasst werden können. Das ist insofern bemerkenswert, da NM IIA nicht das einzige krafterzeugende Motorprotein ist, das in U2OS-Zellen exprimiert wird. Weitere Isoformen der NM-II-Proteine sind vorhanden, scheinen jedoch nicht in der Lage, den Verlust des NM IIA zu kompensieren. Welche Rolle spielen diese Motorproteine dann und was passiert, wenn diese abgeschaltet werden? Man weiß bereits, dass die unterschiedlichen Motorproteine auch unterschiedliche kinetische Eigenschaften besitzen [13]. Bedenkt man, dass eine Kraft nicht nur aufgebaut, sondern auch aufrechterhalten werden muss, ergeben sich hier spannende Fragestellungen, die hoffentlich in der nahen Zukunft entschlüsselt werden können.

\section{Literatur}

[1] Orr AW, Helmke BP, Blackman BR et al. (2006)

Mechanisms of mechanotransduction. Dev Cell 10: 11-20 [2] Janmey PA, Miller RT (2011) Mechanisms of mechanical signaling in development and disease. J Cell Sci 124: 9-18

[3] Choquet D, Felsenfeld DP, Sheetz MP (1997) Extracellular matrix rigidity causes strengthening of integrin-cytoskeleton linkages. Cell 88: 39-48

[4] Brown AE, Discher DE (2009) Conformational changes and signaling in cell and matrix physics. Curr Biol 19: R781-789 [5] Uhler C, Shivashankar GV (2017) Regulation of genome organization and gene expression by nuclear mechanotransduction. Nat Rev Mol Cell Biol 18: 717-727

[6] Finer JT, Simmons RM, Spudich JA (1994) Single myosin molecule mechanics: piconewton forces and nanometre steps. Nature 368: 113-119

[7] Balaban NQ, Schwarz US, Riveline D et al (2001) Force and focal adhesion assembly: a close relationship studied using elastic micropatterned substrates. Nat Cell Biol 3: 466472 
[8] Kamble H, Barton MJ, Jun M et al. (2016) Cell stretching devices as research tools: engineering and biological considerations. Lab Chip 16: 3193-3203

[9] Roca-Cusachs P, Conte V, Trepat X (2017) Quantifying forces in cell biology. Nat Cell Biol 19: 742-751

[10] Hippler M, Weißenbruch K, Richler K et al. (2020) Mechanical stimulation of single cells by reversible hostguest interactions in 3D microscaffolds. Sci Adv 6: 1-13 [11] Hahn C, Mayer F, Thiel M et al. (2019) 3-D Laser Nanoprinting. OPN 30: 28-35

[12] Webster KD, Ng WP, Fletcher DA (2014) Tensional homeostasis in single fibroblasts. Biophys J 107: 146-155 [13] Vicente-Manzanares M, Ma X, Adelstein RS et al. (2009) Non-muscle myosin II takes centre stage in cell adhesion and migration. Nat Rev Mol Cell Biol 10: 778-790
Funding note: Open Access funding enabled and organized by Projekt DEAL. Open Access: Dieser Ar vel wird uncer der Creative Commons Namensnenn Bearbeitung Verbreitung und Wiederge in in Bearbeitung, Verbreitung und sofern Sie den/die ursprünglichen Autor(en) und die Ouelle ordnungsgemäß nennen, einen Link zur Creative Commons Lizenz beifügen und angeben, ob Änderungen vorgenommen wurden. Die in diesem Artikel enthaltenen Bilder und sonstiges Drittmaterial unterliegen ebenfalls der genannten Creative Commons Lizenz, sofern sich aus der Abbildungslegende nichts anderes ergibt. Sofern das betreffende Material nicht unter der genannten Creative Commons Lizenz steht und die betreffende Handlung nich nach gesetzlichen Vorschriften erlaubt ist, ist fur die oben aufgeführten Weiterverwendungen des Materials die Eimwilgung des jeweiligen Demmons. org/licenses/by/4.0/deed de.
Korrespondenzenadresse:

Dr. Kai Weißenbruch

Dr. Marc Hippler

Prof. Dr. Martin Bastmeyer

Zoologisches Institut, Zell- und Neurobiologie

Karlsruher Institut für Technologie (KIT)

Fritz-Haber-Weg 4

D-76131 Karlsruhe

kai.weissenbruch@kit.edu, marc.hippler@kit.edu, martin.bastmeyer@kit.edu https://znbio.zoo.kit.edu

\section{AUTOREN}

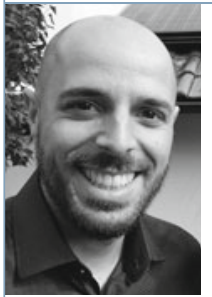

Kai Weißenbruch

2010-2015 Studium der

allgemeinen Biologie, KIT.

2016-2019 Promotion am

Zoologischen Institut, Zell-

und Neurobiologie, KIT. Seit

2020 Postdoc am Zoologi-

schen Institut, Zell- und

Neurobiologie, KIT, mit

Schwerpunkten in Zellbio-

logie und Mechanobiologie.

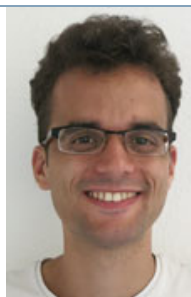

Marc Hippler

2010-2016 Physikstudium am KIT. 2016-2020 Promo-

tion am Institut für Angewandte Physik sowie am Zoologischen Institut, KIT.

Seit 2021 wissenschaftlicher Mitarbeiter am Institut für Nanotechnologie, KIT. Forschungsschwerpunkt in der 3D-Laserlithografie für Anwendungen in den Biowissenschaften.

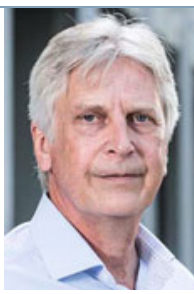

Martin Bastmeyer

Studium der Biologe und Promotion. 1988-1994 Postdoc am Friedrich-

Miescher-Labor in Tübingen und am Salk Institute in San Diego, CA, USA. 1990-1998 Wissenschaftlicher Mitarbeiter an der Universität Konstanz. 1998-2001 HeisenbergStipendium der DFG. 2001-2004 Professor für Neurobiologie an der Universität Jena. Seit 2004 Professor für Zell- und Neurobiologie am KIT und Leiter des Zoologischen Instituts.

\section{Hier steht eine Anzeige.}

\section{Springer}

